\title{
A Rare Case of Parkinson's Disease with Severe Neck Pain Owing to Crowned Dens Syndrome
}

\author{
Teruyuki Takahashi ${ }^{a}$ Masato Tamura, ${ }^{a}$ Keiichi Osabe ${ }^{b}$ \\ Takashi Tamiya $^{c}$ Kenji Miki ${ }^{a, d}$ Mai Yamaguchi ${ }^{a, d}$ Kanno Akira ${ }^{a, d}$ \\ Satoshi Kamei ${ }^{d}$ Toshiaki Takasu $^{\text {a,d }}$ \\ Departments of ${ }^{a}$ Neurology, ${ }^{b}$ Orthopaedic Surgery, and ${ }^{C}$ Psychology, Nagaoka-Nishi \\ Hospital, Nagaoka, and ${ }^{\mathrm{d}}$ Division of Neurology, Department of Medicine, Nihon University \\ School of Medicine, Tokyo, Japan
}

\section{Key Words}

Parkinson's disease - Musculoskeletal pain - Crowned dens syndrome - Odontoid process ·

Cervical computed tomography scanning $\cdot$ Corticosteroid

\begin{abstract}
Background: Pain is regarded as one of the most common nonmotor symptoms in Parkinson's disease (PD). In particular, musculoskeletal pain has been reported as the most common type of PD-associated pain. Crowned dens syndrome (CDS), related to microcrystalline deposition in the periodontoid process, is the main cause of acute or chronic cervical pain. Case Presentation: This report describes the case of an 87-year-old woman who had severe bradykinesia, muscle rigidity, gait disturbance and neck pain. Laboratory examination

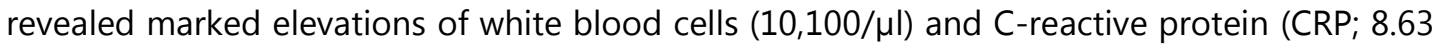
$\mathrm{mg} / \mathrm{dl}$ ). She was primarily diagnosed with severe and untreated PD, corresponding to Hoehn and Yahr scale score IV, with musculoskeletal pain and urinary tract infection. The patient was treated with antiparkinsonism drugs, antibiotic agents and nonsteroidal anti-inflammatory drugs, but they had only limited effects. Cervical plain computed tomography (CT) scanning detected remarkable crown-like calcification surrounding the odontoid process. Based on CT findings, the patient was diagnosed as having CDS with PD, and was immediately treated with corticosteroid. The severe neck rigidity with pain and the serum CRP level $(0.83 \mathrm{mg} / \mathrm{dl})$ of the patient were drastically improved within a week by the additional corticosteroid therapy. Conclusion: Severe neck rigidity and bradykinesia in this patient might have strengthened the chondrocalcinosis around the odontoid process. Cervical plain CT scan is necessary and useful for the definitive diagnosis of CDS. CDS should be considered as a
\end{abstract}


differential diagnosis of a possible etiology for musculoskeletal pain related to rigidity and bradykinesia in PD.

(C) 2014 S. Karger AG, Basel

\section{Background}

Pain in Parkinson's disease (PD) was first described in 1817 by James Parkinson in 'An essay on the shaking palsy' $[1,2]$. Beside the well-known motor symptoms in PD, nonmotor symptoms (NMS), including pain, are commonly experienced in actual clinical practice, but remain insufficiently understood because of their fluctuations and the lack of spontaneous complaints by PD patients [3]. In previous studies, pain was reported as one of the most common NMS in PD, and its prevalence ranged from 40 to $85 \%$ (mean 67.6\%) during disease $[3,4]$. A recent survey evaluating PD patients' perception of their most troublesome symptoms found that pain ranked high in all stages of disease $[2,5]$. Thus, pain in PD is currently regarded as a major cause of reduced health-related quality of life and a more distressing symptom than motor symptoms in some PD patients [2, 4].

Crowned dens syndrome (CDS) is a radioclinical entity defined by the association of microcrystalline deposition in the transverse ligament of the atlas around the odontoid process and periodic acute cervico-occipital pain with fever, neck stiffness and biological inflammatory syndrome [6, 7]. The microcrystalline deposition, most often calcium pyrophosphate dehydrate (CPPD) crystals and/or hydroxyapatite (HA) crystals, can remain asymptomatic or be responsible for chronic neck pain or spinal cord compression [6-10]. In general, CPPD crystal deposition disease is clinically associated with acute episodic mono- or oligo-arthritis, termed 'pseudogout', involving a large joint (including the knees, wrists and ankles) [6-10].

This report describes the rare case of an 87-year-old woman who had severe and untreated PD with severe neck pain owing to CDS. Cervical plain computed tomography (CT) scanning was particularly useful for the diagnosis of CDS. In addition to the antiparkinsonism drugs, the administration of corticosteroid drastically improved the patient's symptoms, particularly severe neck pain, but nonsteroidal anti-inflammatory drugs (NSAID) had only limited effects.

\section{Case Presentation}

On November 5, 2012, an 87-year-old Japanese woman with a history of hypertension was admitted to our hospital because of severe bradykinesia, muscle rigidity, gait disturbance and neck pain. She had noticed motor symptoms, such as slowness, stiffness and difficulty walking, for approximately 2 years, but had undergone no treatment. Her motor symptoms gradually deteriorated, and severe neck and back pain developed from around November 2, after which she became almost bedridden.

At admission, her body temperature was $36.9^{\circ} \mathrm{C}$ and her blood pressure was $124 / 70$ $\mathrm{mm} \mathrm{Hg}$. Chest and abdominal examinations did not show any abnormal findings, but she complained of severe constipation. Upon neurological examination, a remarkable mask-like face and a low voice were shown. She presented marked cogwheel rigidity in both elbows, wrist and knee joints, and lead-pipe rigidity in her neck and trunk, with severe cervical and back pain. Additionally, she demonstrated remarkable bradykinesia, postural instability and severe gait disturbance (short-stepped gait), with freezing phenomenon and pulsion. The tremor at rest was unremarkable. She was oriented and her memory was preserved. There 
Takahashi et al.: A Rare Case of Parkinson's Disease with Severe Neck Pain Owing to Crowned Dens Syndrome

was neither agnosia nor apraxia. Initial routine laboratory examinations revealed marked elevation of white blood cells $(10,100 / \mu \mathrm{l})$ and C-reactive protein (CRP; $8.63 \mathrm{mg} / \mathrm{dl}$ ). The level of serum uric acid $(5.2 \mathrm{mg} / \mathrm{dl})$ was not elevated. Blood examination results for connective tissue disease or vasculitis, such as rheumatoid factor, cytoplasmic (C-) antineutrophil cytoplasmic antibody (ANCA), perinuclear (P-) ANCA, and antinuclear, anticardiolipin, anti-CL- $\beta 2 \mathrm{GPI}$, anti-double-strand DNA and anticyclic citrullinated peptide (CCP) antibodies, were all negative or unremarkable. A large number of white blood cells and bacilli were shown in the urine examination. A brain magnetic resonance imaging (MRI) study demonstrated almost normal findings. The patient was primarily diagnosed with severe and untreated PD with pain and NMS as well as urinary tract infection. Her parkinsonism at the 'off' state corresponded to Hoehn and Yahr scale score IV and the Unified Parkinson's Disease Rating Scale (UPDRS) revealed part I score 1, part II score 23 and part III score 62. The patient was treated with antiparkinsonism drugs (levodopa/benserazide at $100 \mathrm{mg} / 25 \mathrm{mg}$ and droxidopa at $100 \mathrm{mg}$ orally two times daily), antibiotic agents (ceftriaxone sodium hydrate at $2 \mathrm{~g}$ intravenously daily) and NSAID such as loxoprofen sodium hydrate and diclofenac sodium. The patient's parkinsonism, such as marked muscle rigidity of the limbs and trunk, bradykinesia and postural instability, was improved and she could walk with a walker. In particular, the UPDRS parts II and III, which reflect the motor symptoms in PD, markedly declined to scores of 10 and 22, respectively, at the 'on' state by dopaminergic therapy. In addition, dyskinesia and motor fluctuation such as 'wearing off' and 'on-off' phenomena were not recognized at all, so the UPDRS part IV, which reflects the motor complications in PD during dopaminergic therapy, revealed a score of only 1. However, her slight fever $\left(37.7^{\circ} \mathrm{C}\right)$, neck rigidity with severe pain and elevation of the serum CRP level $(10.47 \mathrm{mg} / \mathrm{dl})$ continued. Blood culture, serum procalcitonin and routine cerebrospinal fluid studies all revealed negative results or were within the normal range; thus, meningitis was negative.

On November 10, a cervical plain CT scan detected remarkable crown-like calcification around the odontoid process (fig. 1a-c). A three-dimensional reconstruction image confirmed more remarkable crown-like calcification on the posterior side of the dens (fig. 1d). A cervical MRI study did not demonstrate any direct compression of the cervical cord (data not shown). Based on these cervical CT findings, the patient was diagnosed as having CDS with PD, and was immediately treated with corticosteroid (prednisolone at $30 \mathrm{mg}$ orally daily, tapering off by $5 \mathrm{mg}$ a week). The patient's slight fever, neck rigidity with severe pain and serum CRP level $(0.83 \mathrm{mg} / \mathrm{dl})$ were drastically improved within a week by the course of corticosteroid therapy. She was discharged home on December 11, 2012.

\section{Discussion}

In previous studies, PD-associated pain was categorized into a number of different subtypes [2-4]. Earlier classifications of PD-associated pain tended to emphasize its relationship to dopaminergic therapy including off-period pain, painful dystonic spasms and peak-dose pain [2-4]. Recently, Ford [11] classified five modalities of PD-associated pain: musculoskeletal, radicular-neuropathic, dystonic, central pain and akathisia (table 1). This classification provides a useful framework for managing PD-associated pain in clinical practice $[2,11]$. Beiske et al. [12] described musculoskeletal and dystonic pain as the most common type of pain. The frequency of musculoskeletal pain is estimated to be $70 \%$ in PD patients, whereas dystonic pain has been reported at 40\% [2,12]. PD-associated pain has been reported to occur at various periods during disease [2-4]. In particular, the onset of 
Takahashi et al.: A Rare Case of Parkinson's Disease with Severe Neck Pain Owing to Crowned Dens Syndrome

musculoskeletal pain often precedes the diagnosis of PD or the onset of motor symptoms [24]. Musculoskeletal pain in PD is regarded as resulting from a combination of factors including rigidity, arthralgic pain, skeletal deformity and mechanical factors [2-4]. However, skeletal deformities and arthritic symptoms in PD patients may suggest rheumatoid arthritis, and lead to clinical confusion and inappropriate treatment [2]. In general, muscle rigidity and akinesia are the most common motor symptoms in PD, and often fluctuate due to hyperdopaminergic 'on' or hypodopaminergic 'off' states during anti-PD treatment. Musculoskeletal pain has been regarded as correlating with a hypodopaminergic 'off' state in PD [2]. Therefore, dopaminergic therapy may improve musculoskeletal pain related to rigidity and akinesia, as well as the different type of levodopa-responsive pain such as dystonic pain $[2,11]$. Moreover, a multidirectional approach for pain management, including rehabilitation and classical analgesic agents such as NSAID, has been recommended, and these approaches may also be beneficial to rescue rheumatologic or orthopedic symptoms as a complication of $\mathrm{PD}[2,4,11,12]$.

CDS is defined as a combination of acute feverish cervical pain and calcification in the transverse ligament of the atlas around the odontoid process [6-10,13,14]. In 1985, Bouvet et al. [14] first described and presented the entity of CDS [8-10,13]. The diagnosis of CDS is based on the combination of clinical, biological, radiological and therapeutic findings: acute periodic attacks of feverish cervico-occipital pain and stiffness with biological inflammatory syndrome, radiological identification of crown-like calcification around the odontoid process due to microcrystalline deposits, and drastic improvement of symptoms under treatment with NSAID or colchicine [6-10,13,14]. However, in actual clinical practice, the diagnosis of CDS is often difficult because of its atypical symptoms $[6,7,9,10]$. In particular, the triad of fever, headache and neck stiffness is highly suggestive of infectious meningitis $[6,9,10]$. Patients with symptoms like meningitis are not rare in clinical practice, so CDS should be considered as a differential diagnosis of a possible etiology for fever, headache and neck pain of unknown origin [6, 9, 10]. Moreover, when occipitotemporal, mandible and shoulder girdle pain is associated with inflammatory syndrome, CDS may also mimic polymyalgia rheumatica (PMR) and/or giant cell arteritis (GCA) [6, 10, 15]. Several previous reports described chondrocalcinosis among a wide spectrum of rheumatic diseases because of constitutional symptoms, but not especially in the form of CDS [6, 18-10,13-15]. Thus, some atypical symptoms of CDS may be difficult to distinguish from meningitis, PMR and/or GCA, and may easily lead to a misdiagnosis and an unnecessary lumbar puncture or temporal artery biopsy [6-10, 13-15].

In this report, the patient was an elderly Japanese woman who had severe and untreated PD, corresponding to Hoehn and Yahr scale score IV and UPDRS part III score 62, with severe neck pain and urinary tract infection. Her neck pain was primarily diagnosed as musculoskeletal pain related to rigidity and bradykinesia in PD. Her motor symptoms of PD were improved by dopaminergic therapy, and the UPDRS part III markedly declined to a score of 22. However, the dopaminergic therapy and NSAID, according to the conventional guidelines for the management of pain in PD $[2,4,11,12]$, had only limited effects in this case. Cervical plain CT scan focusing on C-1 and -2 detected a typical half-ringed crown-like form of calcification, which was the most important clinical feature of CDS, behind the dens; this played a much more definitive role for the diagnosis of CDS than cervical MRI study. In addition, a moderate dosage of corticosteroid (prednisolone at $30 \mathrm{mg}$ orally daily) led to drastic improvement of the patient's pathological conditions along with normalization of inflammatory markers. Previous studies have described the rheumatologic or orthopedic conditions in PD with musculoskeletal pain, but CDS as a complication of PD has not been anticipated at all [2-7]. Similarly, previous studies have described the difficulty of distin- 
guishing CDS from meningitis, PMR and/or GCA, but CDS as the cause of musculoskeletal pain in PD has also not been anticipated at all [8-14]. The authors considered that the PD symptoms, such as severe neck rigidity and bradykinesia, in this patient might have strengthened the chondrocalcinosis, including CPPD and/or hydroxyapatite microcrystalline depositions, in periarticular structures around the odontoid process. Thus, CDS should be considered as a differential diagnosis of a possible etiology for musculoskeletal pain, particularly neck pain, related to rigidity, akinesia and/or bradykinesia in PD. Currently, cervical plain CT scanning is regarded as the gold standard of CDS diagnosis, so it should be more actively performed in PD patients with severe neck rigidity and pain. Moreover, the active use of a moderate dosage of corticosteroid may be recommended for musculoskeletal pain, particularly neck pain, with PD, in addition to the conventional first-line therapy by levodopa and NSAID.

To our knowledge, there have been no reports of PD patients with CDS as a complication. In previous studies of PD-associated pain, including musculoskeletal pain, CDS might have been hidden. Therefore, the authors consider that it is important to report this patient as the first case of PD with severe neck pain owing to CDS. At present, CDS is regarded as a rare neurological condition, but more cases of CDS may be detected in PD patients with musculoskeletal pain by actively performing cervical plain CT scanning. Therefore, further research and an accumulation of cases like that in our report are necessary to obtain a good understanding of the correlation of CDS and musculoskeletal pain in PD.

\section{Acknowledgement}

The authors are grateful to the patient for the permission to publish this information.

\section{Disclosure Statement}

The authors declare that they have no competing interests. There are no financial conflicts of interest in this study.

\section{References}

1 Parkinson J: An essay on the shaking palsy. 1817. J Neuropsychiatry Clin Neurosci 2002;14:223-236.

2 Ha AD, Jankovic J: Pain in Parkinson's disease. Mov Disord 2012;27:485-491.

-3 Bonnet AM, Jutras MF, Czernecki V, Corvol JC, Vidailhet M: Nonmotor symptoms in Parkinson's disease in 2012: relevant clinical aspects. Parkinsons Dis 2012;2012:198316.

-4 Truini A, Frontoni M, Cruccu G: Parkinson's disease related pain: a review of recent findings. J Neurol 2013;260:330-334.

5 Politis M, Wu K, Molloy S, G Bain P, Chaudhuri KR, Piccini P: Parkinson's disease symptoms: the patient's perspective. Mov Disord 2010;25:1646-1651.

-6 Aouba A, Vuillemin-Bodaghi V, Mutschler C, De Bandt M: Crowned dens syndrome misdiagnosed as polymyalgia rheumatica, giant cell arteritis, meningitis or spondylitis: an analysis of eight cases. Rheumatology 2004;43:1508-1512.

7 Constantin A, Bouteiller G: Acute neck pain and fever as the first manifestation of chondrocalcinosis with calcification of the transverse ligament of the atlas. Five case-reports with a literature review. Rev Rhum Mal Osteoartic Engl Ed 1998;65:582-585.

$>8$ Wu DW, Reginato AJ, Torriani M, Robinson DR, Reginato AM: The crowned dens syndrome as a cause of neck pain: report of two new cases and review of the literature. Arthritis Rheum 2005;53:133-137.

-9 Sato Y, Yasuda T, Konno S, Kuwayama A, Komatsu K: Pseudogout showing meningoencephalitic symptoms: crowned dens syndrome. Intern Med 2004;43:865-868. 


DOI: $10.1159 / 000362514$

(C) 2014 S. Karger AG, Base www.karger.com/crn

Takahashi et al.: A Rare Case of Parkinson's Disease with Severe Neck Pain Owing to Crowned Dens Syndrome

10 Takahashi T, Minakata Y, Tamura M, Takasu T, Murakami M: A rare case of crowned dens syndrome mimicking aseptic meningitis. Case Rep Neurol 2013;5:40-46.

11 Ford B: Pain in Parkinson's disease. Clin Neurosci 1998;5:63-72.

12 Beiske AG, Loge JH, Rønningen A, Svensson E: Pain in Parkinson’s disease: prevalence and characteristics. Pain 2009;141:173-177.

13 Baysal T, Baysal O, Kutlu R, Karaman I, Mizrak B: The crowned dens syndrome: a rare form of calcium pyrophosphate deposition disease. Eur Radiol 2000;10:1003-1005.

14 Bouvet JP, le Parc JM, Michalski B, Benlahrache C, Auquier L: Acute neck pain due to calcifications surrounding the odontoid process: the crowned dens syndrome. Arthritis Rheum 1985;28:1417-1420.

15 Gonzalez-Gay MA, Garcia-Porrua C, Llorca J, Gonzalez-Louzao C, Rodriguez-Ledo P: Biopsy-negative giant cell arteritis: clinical spectrum and predictive factors for positive temporal artery biopsy. Semin Arthritis Rheum 2001;30:249-256.

Table 1. Classification of pain and discomfort in PD $[2,4,11]$

\begin{tabular}{ll}
\hline Types of pain/discomfort & Features \\
\hline Musculoskeletal & $\begin{array}{l}\text { Aching and cramping pain, frozen shoulder, back pain } \\
\text { May be caused by parkinsonian rigidity, immobility and mechanical factors } \\
\text { Associated rheumatologic and orthopedic disease }\end{array}$ \\
\hline Dystonic & $\begin{array}{l}\text { Dystonic posturing and spasms } \\
\text { Levodopa-induced dystonia: wearing off dystonia or morning dystonia, } \\
\text { peak-dose dystonia, diphasic dystonia }\end{array}$ \\
\hline Neuropathic & $\begin{array}{l}\text { Radicular neuropathic: localized to a specific nerve root distribution of } \\
\text { dermatome } \\
\text { Probably not related to PD } \\
\text { Peripheral neuropathic: symmetrical and distal }\end{array}$ \\
\hline Central & Poorly localized \\
& Boring, constant, burning \\
& Vague sensations of tension and discomfort \\
& Visceral and autonomic discomforta \\
\hline Akathitic discomfort & Inner restlessness, urge to move \\
\hline Others & Oral and genital pain: burning mouth or vagina syndrome \\
& May represent sensory wearing off and may improve with levodopaa \\
\hline
\end{tabular}

a May be considered a nonmotor 'off' symptom. 


\section{Case Reports in Neurology}
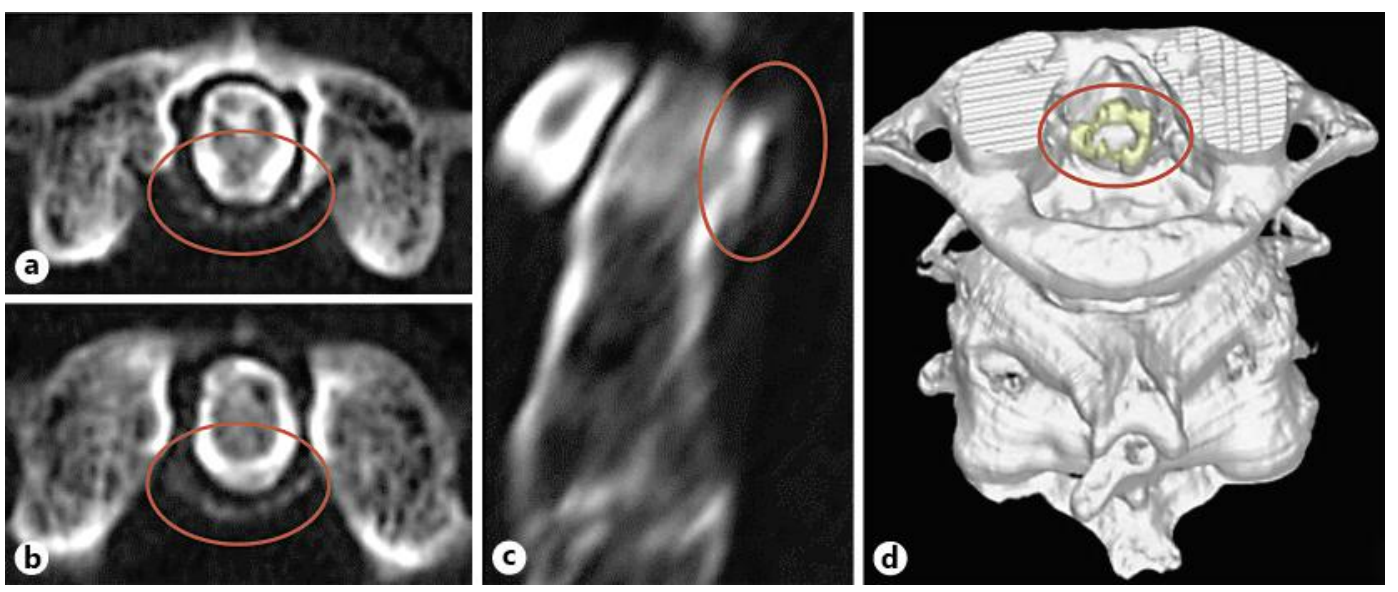

Fig. 1. Cervical plain CT scanning images surrounding the odontoid process (dens). a, $\mathbf{b}$ The axial image demonstrates the clear half-ringed form of calcification at the posterolateral side of the dens. c The sagittal image demonstrates the linear calcification at the posterior side of the dens. d A threedimensional reconstruction obtained from data of a cervical plain CT demonstrates the marked crownlike calcification at the posterior side of the dens. The diagnosis of CDS was confirmed by these cervical CT findings.

Takahashi et al.: A Rare Case of Parkinson's Disease with Severe Neck Pain Owing to Crowned Dens Syndrome
(C) 2014 S. Karger AG, Basel www.karger.com/crn 